\title{
Nonlinear deformation of circular discrete ribbed plate under influence of pulse loading
}

\author{
Rustam Khalmuradov ${ }^{*}$, Utkir Nishonov \\ Samarkand State University, Samarkand, Uzbekistan
}

\begin{abstract}
The stress-strain state of a circular disc, discretely finned in a circle, under the influence of the pulse loading, is numerically investigated. Thus the geometric nonlinearity between displacement and deformation is taken into account. The structure consists of boarding and reinforced ribs, the materials of which are the same and obey Hooke's law. The sections of the ribs are constant. The height of the ribs and their locations are specified using a unit function. It is considered that the plate is deformed under the influence of the pulsed loading. A numerical method is used to solve the problem.
\end{abstract}

\section{Introduction}

In modern technology and construction, thin elastic structures and their elements are widely used. These elements are often subjected to various dynamic loads and operate under high strain rates and in other difficult operating conditions [1]. To increase the overall rigidity of the structure in such conditions, its thin-walled part is reinforced by ribs. Slightly increasing the structure's weight, they increase its strength and are indispensable for the transfer of forces close to concentrated. At the same time, the material consumption of structures is reduced without reducing their carrying capacity, which is beneficial both in design and in economic terms. Therefore, special attention is paid to determining the strength characteristics of plates of increased rigidity under the action of compressive loads in studies of the dynamic behavior of plates reinforced with stiffeners [2].

In solving problems on the vibrations of plates reinforced with ribs, two different methods are used, depending on how often the ribs are located-sufficiently tightly or less frequently [3]. A comparison of these methods was made for two types of the location of the edges and indicated that the best results that coincide with the experiment are obtained by solving the problem as contact and taking into account the discrete nature of the reinforcements. It should be mentioned that the complexities of analytical calculations forced authors to confine themselves to relatively simple configurations of ribbed plates. Therefore, by now, in the scientific literature, a small number of papers have concerned the analytical calculation of ribbed plates [4-6].

In dynamic calculations of ribbed plates, an important role is to investigate natural oscillations since reinforcements change the frequency spectrum and the shape of the oscillations [7]. The application of numerical methods, particularly the method of finite

\footnotetext{
*Corresponding author: rustami@list.ru
} 
differences, to solve dynamic problems supported by ribs of rectangular and circular plates was considered in $[9,10]$. Numerical calculations of the required parameters are a relatively new and promising direction in studies of the behavior of ribbed and layering structural elements and, in particular, plates [11-13]. Ideas that underlie some numerical developments are related to the spectral finite element or wave methods of finite It elements [14-16]. Analysis of some scientific papers devoted to the subject of numerical calculations of various aspects various of ribbed structures is given in [17].

Investigations by dynamic and experimental methods of the deformed and stressed state of shells and plates [8-21] are of great importance. In the paper [22], based on the geometrically nonlinear theory of deformation of Mindlin - Reissner shells, the stress-strain state of shallow shell structures of double curvature, reinforced by the concavity side with a different number of edges, is analyzed. The work [33] is devoted to determining the stressstrain state of a ribbed spherical dome under the action of a pulsed force. The work [23] is devoted to determining the boundary conditions for contacting cylindrical shells and plates.

Despite numerous publications, and in connection with the continuous expansion of the field of applicability of ribbed plates and shells, the problem of formulating more refined models $[25,26]$, describing the dynamic processes in them and searching for effective analytical methods for solving the corresponding initial-boundary value problems of mathematical physics with allowance for nonlinear properties, such as boarding and ribs. Based on this, the article is devoted to the analytical calculation of a round plate reinforced by ring stiffeners and a generalization of the results of $[24,26]$ to the case of taking into account the discrete number of edges. It can be extended to generalize the results $[24,26]$, in the case of taking into account a discrete number of ribs.

\section{Methods}

\subsection{Statement of the problem}

In the cylindrical coordinate system $(r, \varphi, z)$, a circular plate is clamped along the edges and reinforced by annular ribs of rigidity. In this case, the axis $z$ is perpendicular to the plane of the plate. It is considered that the structure consists of boarding and reinforced ribs, the materials of which are the same and are subject to Hooke's law. The ribs have quadrangular cross-sections and are attached to the inner surface of the plate. Vibrations of the plate are excited by pulse loading on the outer surface of the plate. The cross-sections of the ribs are the same and constant along the radial and angular coordinates. The height of ribs and their location $H\left(r_{i}\right)$ are determined using unit functions $\delta\left(r-r_{i}\right)$ [3]:

$$
H(r)=\sum_{i=1}^{m} h^{i} \delta\left(r-r_{i}\right) ; \delta\left(r-r_{i}\right)= \begin{cases}0, & r<a_{i}, r>b_{i} \\ 1, & a_{j}<r<b_{i}\end{cases}
$$

where: $a_{i}=c_{i} / 2 ; b_{i}=r_{j}+c_{i} / 2 ; r_{i}$ are the coordinate of the midpoint of the contact zone of the sheeting and the $i$-th rib; $h^{i}(r), c_{i}$ are height and width of the $i$-th rib; $m$ is number of ribs. 


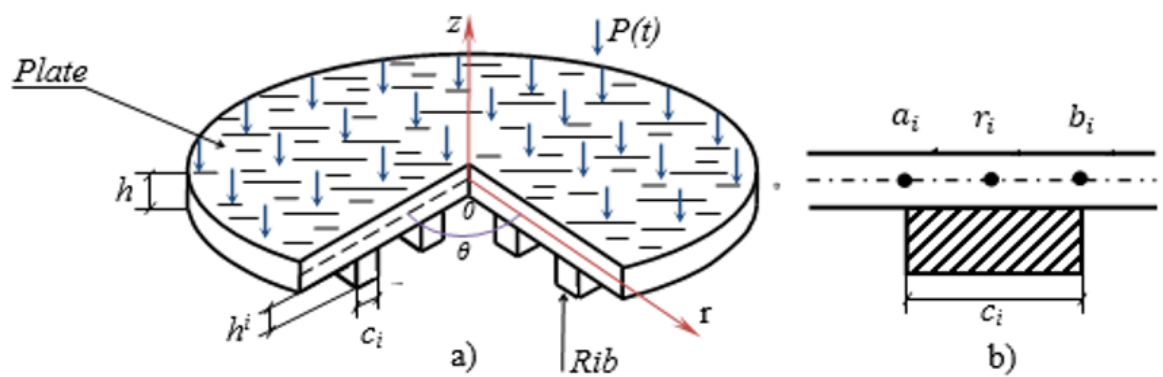

Fig. 1. General type of the ribbed plate (a) and cross-section rib (b).

The equations of the nonlinear theory of vibration of plates of the Timoshenko type are accepted as resolving. For axisymmetric problems, these equations can be written as [3]:

$$
\begin{gathered}
\left(N_{1} r\right)^{\prime}-N_{2}=r \rho[\ddot{u}(h+F)+\ddot{\psi} S] ;(r Q)^{\prime}+\left(N_{1} r w^{\prime}\right)^{\prime}=r \rho \ddot{w}(h+F)-r P \\
\left(r M_{1}\right)^{\prime}-M_{2}-r Q=r \rho\left[\ddot{\psi}\left(h^{3} / 12+J\right)+\ddot{u} S\right]
\end{gathered}
$$

where, $\rho, h$ are the density and thickness of the skin, respectively;

$$
F=\sum_{i=1}^{m} F^{i}(r) \delta\left(r-r_{i}\right) ; S=\sum_{i=1}^{m} S^{i}(r) \delta\left(r-r_{i}\right) ; J=\sum_{i=1}^{m} J^{i}(r) \delta\left(r-r_{i}\right)
$$

$F^{j}(r), S^{i}(r), J^{i}(r)$ are accordingly, the cross-sectional area of the $i-$ rib element, with a width equal to unity and height $h^{i}(r)$ in the section with $r=$ const, is the static moment and moment of inertia of this section relative to the coordinate axis; $P(t)$ is external load function

The boundary conditions for the considered design of the plate relative to the movements are:

a) conditions of hard pinching at the edges of the plate

$$
u=w=\psi=0
$$

b) symmetry conditions in the center of the plate

$$
u=\frac{\partial w}{\partial r}=\psi=0 \text { at } r=0
$$

The initial conditions are characterizing the state of the plate at $t=0$ zero.

\subsection{Mathematical model}

Displacements of an arbitrary point of the normal to the middle surface of a plate with coordinate $z$ are equal 


$$
\boldsymbol{u}^{z}=\boldsymbol{u}+z \boldsymbol{\psi}, \quad \boldsymbol{w}^{z}=\boldsymbol{w}
$$

and taking into account of axisymmetric of loading $v^{z}=v=0$, where $u, v, w$ - the displacement of the points of the middle surface of the plate along the coordinate axes; $\psi$ the angle of rotation of the normal segment to the middle surface of the plate.

The extensional strain in the middle surface is taken the form [25]

$$
\varepsilon_{1}=\frac{\partial u}{\partial r}-\frac{w}{R}+\frac{1}{2}\left(\frac{\partial w}{\partial r}\right)^{2} ; \varepsilon_{2}=u / r-w / R
$$

the deformations of an arbitrary point along the thickness of the plate have the form

$$
\varepsilon_{1}^{z}=\varepsilon_{1}+z \frac{\partial \psi}{\partial r} ; \quad \varepsilon_{2}^{z}=\varepsilon_{2}+z \frac{\psi}{r}
$$

The relationship between the stresses acting at the boarding points and deformations is written as follows

$$
\sigma_{1}^{0}=\frac{E}{1-\mu^{2}}\left(\varepsilon_{1}^{z}+\mu \varepsilon_{2}^{z}\right) ; \sigma_{2}^{0}=\frac{E}{1-\mu^{2}}\left(\varepsilon_{2}^{z}+\mu \varepsilon_{1}^{z}\right) ; \sigma_{13}^{0}=\frac{E}{2(1+\mu)} \varepsilon_{13}^{z}
$$

where $\varepsilon_{13}^{z}=f(z)\left(\frac{\partial W}{\partial r}+\psi\right) ; E, \mu$ are the modulus of elasticity and Poisson's ratio of the plate material; $f(z)$ is a function characterizing the law of stress distribution $\sigma_{13}^{O}$ over plate thickness $f(z)=f_{0}(z)$ or the smooth part of the plate; $f(z)=f_{l}(z)$ at the points where the ribs are located $[3,26]$

$$
f_{o}(z)=6\left[0.25-\left(\frac{z}{h}\right)^{2}\right] ; \quad f_{1}(z)=\frac{3 h(h+2 H)}{2(h+H)^{2}}\left(1+2 \frac{z}{h}\right)\left(1-2 \frac{z}{h+2 H}\right)
$$

$H$ is the height of the rib.

Forces and moments per unit length of the section, acting in the cross-section of the plate, supported by the ribs, have the following form [22]:

$$
N_{1}=N_{1}^{0}+N_{1}^{R} ; \quad N_{2}=N_{2}^{0}+N_{2}^{R} ; M_{1}=M_{1}^{0}+M_{1}^{R} ; M_{2}=M_{2}^{0}+M_{2}^{R} ; Q=Q^{0}+Q^{R}
$$

Here the index "1" refers to the radial and "1" to tangential directions.

Forces and moments related to the boarding have forms:

$$
\begin{gathered}
N_{1}^{0}=\int_{-h / 2}^{h / 2} \sigma_{1}^{0} d z=\frac{E h}{1-\mu^{2}}\left(\varepsilon_{1}+\mu \varepsilon_{2}\right)=\frac{\partial \psi}{\partial r}+\mu \frac{\psi}{r} ; \\
N_{2}^{0}=\int_{-h / 2}^{h / 2} \sigma_{2}^{0} d z=\frac{E h}{1-\mu^{2}}\left(\varepsilon_{2}+\mu \varepsilon_{1}\right)=\frac{\psi}{r}+\mu \frac{\partial \psi}{\partial r} ; \\
M_{1}^{0}=\int_{-h / 2}^{h / 2} \sigma_{1}^{0} z d z=\frac{E h^{3}}{12\left(1-\mu^{2}\right)}\left(\frac{\partial \psi}{\partial r}+\mu \frac{\psi}{r}\right) ; M_{2}^{0}=\int_{-h / 2}^{h / 2} \sigma_{2}^{0} z d z=\frac{E h^{3}}{12\left(1-\mu^{2}\right)}\left(\frac{\psi}{r}+\mu \frac{\partial \psi}{\partial r}\right) ; \\
Q^{0}=\int_{-h / 2}^{h / 2} \sigma_{13}^{0} d z=\frac{5}{6} \frac{E h}{2(1+\mu)} \varepsilon_{13}^{z} . D=\frac{E h^{3}}{12\left(1-\mu^{2}\right)} ;(5)
\end{gathered}
$$


To determine the forces, moments, and shearing forces acting in the cross-sections of the ribs, the whole structure is considered as a step-variable plate of thickness.

Stresses acting into ribs will be determined by the following formulas:

$$
\begin{gathered}
\sigma_{1}^{R}=G\left(\varepsilon_{1}^{z}+\mu \varepsilon_{2}^{z}\right) ; \quad \sigma_{2}^{R}=G\left(\varepsilon_{2}^{z}+\mu \varepsilon_{1}^{z}\right) ; \quad \sigma_{13}^{R}=G_{13} \varepsilon_{13}^{z} \\
G=\frac{E}{1-\mu^{2}} ; \quad G_{13}=\frac{5}{12} \frac{E}{(1+\mu)}
\end{gathered}
$$

where $E, \mu, G_{13}$ are elastic constants of the material of the ribs (we assume that $G_{13}$ includes $k^{2}$ ).

Forces and moments acting on the cross-sections of the ribs have the following form:

$$
\begin{gathered}
M_{1}^{R}=\int_{h / 2}^{h / 2+H} \sigma_{1}^{R} z d z=B\left(\varepsilon_{1}+\mu \varepsilon_{2}\right)+C\left(\frac{\partial \psi}{\partial r}+\mu \frac{\psi}{r}\right) \\
M_{2}^{R}=\int_{h / 2}^{h / 2+H} \sigma_{2}^{R} z d z=B\left(\varepsilon_{2}+\mu \varepsilon_{1}\right)+C\left(\frac{\psi}{r}+\mu \frac{\partial \psi}{\partial r}\right) ; Q^{R}=\int_{h / 2}^{h / 2+H} \sigma_{13}^{R} d z=D_{13}\left(\frac{\partial w}{\partial r}+\psi\right) ; \\
A=G F ; B=G S ; C=G J ; D_{13}=\mathrm{G}_{13} \mathrm{H}\left(\mathrm{r}_{\mathrm{i}}\right)
\end{gathered}
$$

\subsection{Numerical solution method}

To solve the problem (1) - (6), the finite difference method is applied. The scheme for the numerical solution of the problem using this method is based on determining the displacements and angles of rotation at the grid nodes and the deformations, forces, moments, and transverse forces at the center of the element.

To approximate the equations of motion (1), which are centered at the nodal points, the central differences are used

$$
\left[\frac{\partial f}{\partial r}\right]_{i}=\frac{f_{i+1 / 2}-f_{i-1 / 2}}{\Delta r_{i+1 / 2}}
$$

Coordinate derivatives of functions are approximated in increments $\Delta r_{i}=r_{i}-r_{i-1}$. Derivatives with respect to time are approximated by expressions of the form

$$
\left[\frac{\partial^{2} w}{\partial t^{2}}\right]_{i}^{n}=\frac{1}{\tau^{2}}\left[w_{i}^{n+1}-2 w_{i}^{n}+w_{i}^{n-1}\right]
$$

where $\tau$ is the time step, $n$ is the index specifying the time layer.

The resulting finite-difference analog of the system (1) has the form

$$
u_{i}^{n+1}=2 u_{i}^{n}-u_{i}^{n-1}+\tau^{2}\left\{U_{i}^{n} / a-b \times\left(\psi_{i}^{n}-b U_{i}^{n} / a\right) /\left(a c-b^{2}\right)\right\}
$$




$$
\begin{gathered}
w_{i}^{n+1}=2 w_{i}^{n}-w_{i}^{n-1}+\tau^{2} W_{i}^{n} / a \\
\psi_{i}^{n+1}=2 \psi_{i}^{n}-\psi_{i}^{n-1}+\tau^{2} \times\left(\Psi_{i}^{n}-b U_{i}^{n}\right) / a /\left(c-b^{2} / a\right)
\end{gathered}
$$

where: $a=\rho(h+F), b=\rho S, c=\rho\left(h^{3} / 12+J\right)$;

$$
\begin{gathered}
U_{i}^{n}=\frac{\left(r N_{1}\right)_{(i+1 / 2)}^{n}-\left(r N_{1}\right)_{(i-1 / 2)}^{n}}{\Delta r_{i+1 / 2} r_{i+1 / 2}}+\frac{\left(N_{2}\right)_{(i+1 / 2)}^{n}-\left(N_{2}\right)_{(i-1 / 2)}^{n}}{2 r_{i+1 / 2}} ; \\
W_{i}^{n}=\frac{(r Q)_{(i+1 / 2)}^{n}-(r Q)_{(i+1 / 2)}^{n}+\left(\frac{w_{i}^{n}-w_{i-1}^{n}}{\Delta r_{i}}\right) \frac{1}{\Delta r_{i+1 / 2} r_{i+1 / 2}} \times \frac{\left(r N_{1}\right)_{(i+1 / 2)}^{n}-\left(r N_{1}\right)_{(i-1 / 2)}^{n}}{\Delta r_{i+1 / 2}}+}{+\frac{w_{i+1}^{n}-2 w_{i}^{n}+w_{i-1}^{n}}{\left(\Delta r_{i}\right)^{2}} \frac{\left(N_{1}\right)_{(i+1 / 2)}^{n}+\left(N_{1}\right)_{(i-1 / 2)}^{n}+P_{i+\frac{1}{2}}^{n} ;}{2}} \\
\Psi_{i}^{n}=\frac{\left(r M_{1}\right)_{i+1 / 2}^{n}-\left(r M_{1}\right)_{i-1 / 2}^{n}}{\Delta r_{i+1 / 2} r_{i+1 / 2}}-\frac{\left(M_{2}\right)_{i+1 / 2}^{n}+\left(M_{2}\right)_{i-1 / 2}^{n}}{2 r_{i+1 / 2}}-\frac{Q_{i+1 / 2}^{n}+Q_{i-1 / 2}^{n}}{2}
\end{gathered}
$$

From relations (2) and (3) we obtain finite-difference expressions for calculating deformations

$$
\begin{gathered}
\left(\varepsilon_{1}^{z}\right)_{i+1 / 2}^{n}=\left(\varepsilon_{1}\right)_{i+1 / 2}^{n}+z \frac{\psi_{i+1}^{n}-\psi_{i}^{n}}{\Delta r_{i+1 / 2}} ;\left(\varepsilon_{2}^{z}\right)_{i+1 / 2}^{n}=\left(\varepsilon_{2}\right)_{i+1 / 2}^{n}+z \frac{\psi_{i+1}^{n}+\psi_{i}^{n}}{2 r_{i+1 / 2}} \\
\left(\varepsilon_{2}\right)_{(i+1 / 2)}^{n}=\frac{u_{i+1}^{n}+u_{i}^{n}}{2 r_{i+1 / 2}} \\
\left(\varepsilon_{13}^{z}\right)_{i+1 / 2}^{n}=f(z)\left(\frac{w_{i+1}^{n}-w_{i}^{n}}{\Delta r_{i+1 / 2}}+\frac{\psi_{i+1}^{n}+\psi_{i}^{n}}{2}\right) \\
\left(\varepsilon_{1}\right)_{(i+1 / 2)}^{n}=\frac{u_{i+1}^{n}-u_{i}^{n}}{\Delta r_{i+1 / 2}}+\frac{1}{2}\left(\frac{w_{i+1}^{n}-w_{i}^{n}}{2 \Delta r_{i+1 / 2}}\right)^{2}
\end{gathered}
$$

The values of stresses, forces, moments, and cutting forces are found by formulas (4) - (7) using expressions (9).

The formulas in (8) are related only to the internal points of the computational domain. To determine the values of the functions at the boundary points, we use the boundary conditions a) and b) described in the previous section. The hard jamming conditions are approximated exactly, and the symmetry conditions are written in finite differences using the following expression:

$$
\left[\frac{\partial w}{\partial r}\right]_{1}^{n}=\frac{3 w_{1}^{n}-4 w_{2}^{n}+w_{3}^{n}}{2 \Delta r_{2}}
$$

The grid functions on two adjacent time layers, necessary to start the calculations, give the initial conditions, from which we obtain equalities of the form 


$$
w_{i}^{1}=w_{i}^{2}=0
$$

By the above mentioned equalities, it can be found $u_{i}{ }^{n}, w_{i}{ }^{n}, \psi_{i}^{n}$ for any point in time and any point of the difference grid.

The general discrepancy in the approximation of the considered boundary value problem by difference relations does not exceed $O\left(\Delta r^{2}+\tau^{2}\right)$. As the grid steps tend to zero, the residuals also tend to zero. Therefore, difference equations approximate the original differential equations. The sighting values of the grid steps ensuring the stability of the calculations are found from the Courant condition $\tau_{1} \leq \Delta r / c_{1}$ where $c_{1}=\sqrt{E / \rho\left(1-\mu^{2}\right)}$.

\section{Results and Discussions}

The solution of the system of equations (1) calculated deflection, longitudinal forces, shear force, and moments in the sections of rigidly clamped ribbed circular plate under the influence of the uniformly distributed load $P=P_{0} e^{-t / \alpha}$, at $P_{0}=2.5 \mathrm{MPa} ; \alpha=10^{-3} \mathrm{~s}$. Geometric and elastic characteristics of the plate are as follows: $R_{0}=0.5 \mathrm{~m} ; h=0.01 \mathrm{~m}$; $E=75600 \mathrm{MPa} ; \mu=0.3 ; \rho=2640 \mathrm{~kg} / \mathrm{m}^{3}$. When the plate has one annular rib with height $h^{p}=4 \cdot 10^{-2} \mathrm{~m}$ and width $\xi=3.33 \cdot 10^{-2} \mathrm{~m}$ was considered in the here cases are considered where the plate is supported by two and four annular ribs. For comparison, the calculation of the above parameters was performed in a non-reinforced plate (boarding). The results are shown in Fig. 1-5. The case is considered when the plate is supported by two ribs of height $h^{p}=4 \cdot 10^{-2} \mathrm{~m}$ and width $\xi=3.33 \cdot 10^{-2} \mathrm{~m}$. We consider five variants of the location of the ribs with $d=0.2$; 0.4. 2) $d=0.2$; 0.8. 3) $d=0.4$; 0.8. 4) $d=0.4$; 0.6. 5) $d=0.6$; 0.8.

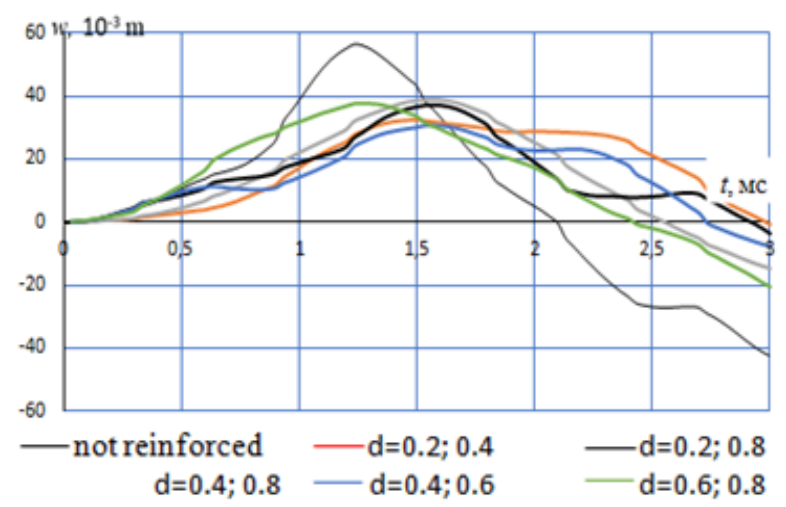

Fig. 1. Deflections of the central point unreinforced and reinforced by two ribs of the plate with height $h^{p}=4 \cdot 10^{-2} \mathrm{~m}$, and $\xi=3.33 \cdot 10^{-2} \mathrm{~m}$.

The results of calculating the deflection of a central point that is not reinforced and supported by two ribs of the plate are shown in Fig. 1 as graphs versus time $0<t<0.003 s$. From these graphs, it follows that the maximum value of the deflection of the central point of the plate, regardless of reinforcement, is achieved in the time interval $0.001<t<0.002$. Thus, the deflection of the central point of the unsupported plate exceeds by at least $37 \%$ the value of the deflection of the central point of the reinforced plate. In the most effective case, when the ribs are located at distances $d=0.4$ and $d=0.6$ from the central point, the presence of ribs leads to a decrease in the deflection value to $44 \%$ (Fig. 1). In other cases of 
reinforcement, the decrease in deflection values is in the range from $37 \%$ to $44 \%$. Hence it follows that the ribs should be supported in the middle of the plate radius.

Figure 2 shows the time dependences of the deflections of the central point of the plate for non-reinforced and reinforced with one, two, and four annular ribs installed at distances $d$ The ribs have the same dimensions: height $h^{p}=4 \cdot 10^{-2} \mathrm{~m}$ and width $\xi=3.33 \cdot 10^{-2} \mathrm{~m}$. The following cases were calculated:

- one rib located at a distance at $d=0.2$. (near the pole);

- two ribs are located near the center and near to the edge of the plate with coordinates $d=0.2 ; 0.8$;

- four ribs are located evenly along the radius of the reference circuit, and the distance between them is $0.2 r$.

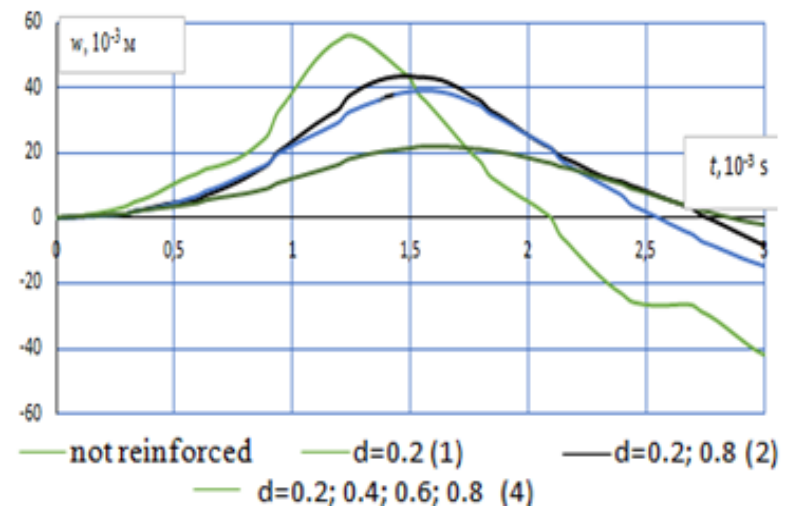

Fig. 2. Deflections of the central point of the unreinforced and reinforced plates with one, two, and four ribs

From these graphs, it follows that the presence of edges leads to a decrease in the maximum values of deflection:

a) in the presence of one rib to $12.5 \%$;

b) in the presence of two ribs up to $40 \%$;

c) in the presence of four ribs up to $57 \%$;

In figure 3 is shown the dependence of the radial force $N_{l}$ from the coordinate $r$ at a fixed value of time $t$ and various $d$. It can be seen that for a given moment in time, the reinforcement of the plate with one rib $(d=0.2)$ leads to an increase of $N_{l}$ up to $55 \%$ compared to the smooth plate. Reinforcement of the plate with two $(d=0.2 ; 0.8)$ ribs reduces the force $N_{l}$ to $55 \%-58 \%$, but reinforcement with four ribs lowers the values $N_{l}$ to almost zero.

In figure 4 the force dependences $N_{2}$ (in a direction perpendicular to the radial direction) from the radial coordinate at $t=6 \cdot 10^{-4} \mathrm{~s}$ and different values of the coefficient $d$. From the figure, we can see a stepwise change of force, and its value is less, the more the number of ribs. The reinforcement of such plate with four annular ribs practically nullifies the influence of the force, except for the attachment points of the ribs $\left[r_{0}=0.1 m(d=0.2)\right.$; $\left.r_{0}=0.2 m(d=0.4) ; r_{0}=0.3 m(d=0.6) ; r_{0}=0.4 m(d=0.8) ;\right]$, where abrupt changes in the values of force are observed. This corresponds to the absence of deflection at this point or its smaller value than neighboring points. This fact is an important factor of the stressed-strain state of the ribbed plate and fully corresponds to the physical nature of the problem. 


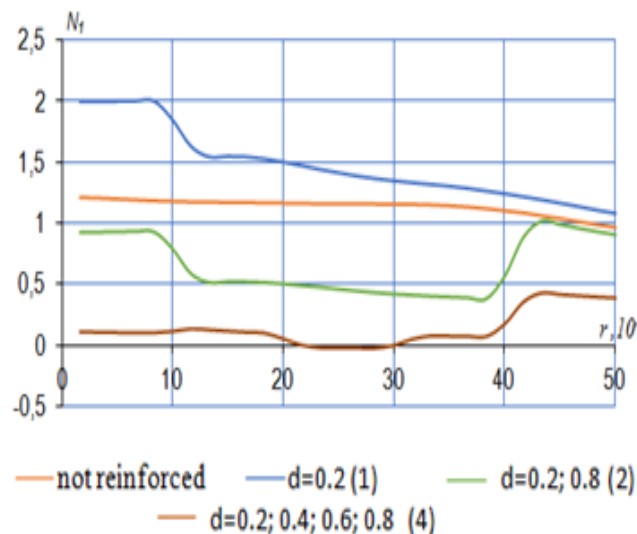

Fig. 3. The dependence of the force $N_{1}$, on the radial coordinate at $t=6 \cdot 10^{-4} \mathrm{~s}$ and different values of the coefficient $d$.

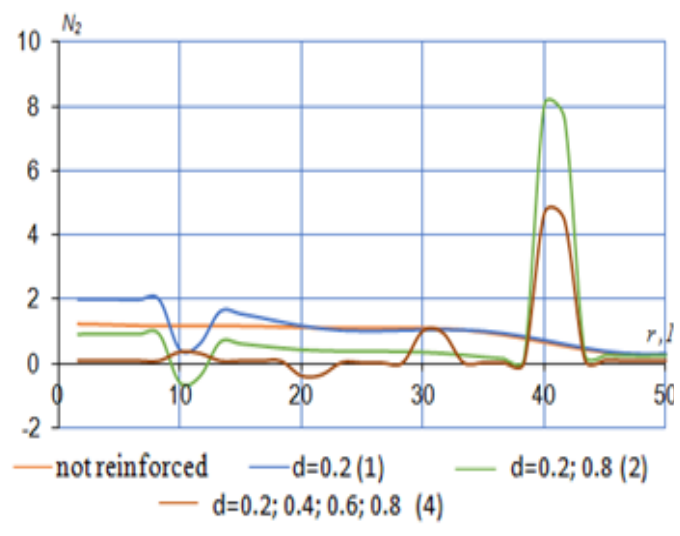

Fig. 4. The dependence of the force $N_{2}$, on the radial coordinate at $t=6 \cdot 10^{-4} \mathrm{~s}$ and different values of the coefficient $d$.

In figure 5, curves for the variation in the shear force $Q$ are plotted as a function of the radial coordinate at $t=6 \cdot 10^{-4} \mathrm{~s}$ and different coefficient values $\alpha$. The shear force at the attachment points of the ribs $(d=0.2 ; 0.6 ; 0.8)$ takes values close to zero. For $r=0.3 m$, all curves $Q$, regardless of the presence and number of reinforcing ribs or their absence, change their sign to the opposite. This indicates that the points of the plate with the coordinate $r>0.3 m$ receive a deflection in the direction opposite to the deflection direction at $r<0.3 \mathrm{~m}$. The amplitude of the shear force $Q$ for a smooth plate varies according to the law close to the sinusoidal one. When the plate is reinforced with stiffening ribs, this pattern is violated. In some cases, it is possible to observe about the same regularity (the plate is supported by four ribs), and in other cases ( $d=0.2$; i.e., the plate is supported by one rib), this regularity is absent.

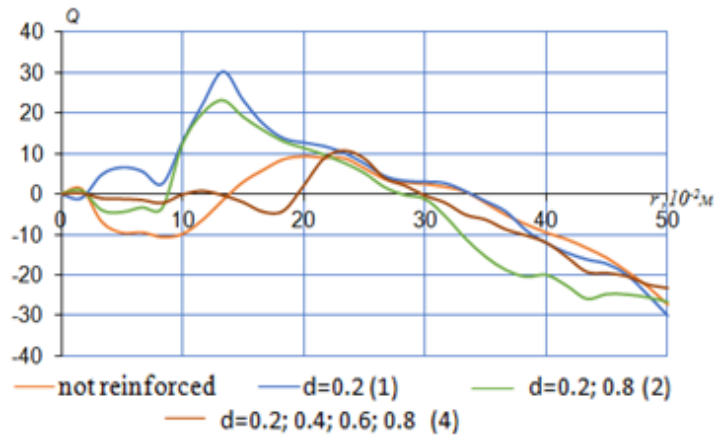

Fig. 4. The dependence of the force $Q$, on the radial coordinate at $t=6 \cdot 10^{-4} \mathrm{~s}$ and different values of the coefficient $d$.

\section{Conclusions}

Here, the numerical solution of the problem and the analysis of the results obtained make it possible to draw some conclusions on the effectiveness of reinforcement of plates.

The results of calculating the deflection of the central point in the case of reinforcement of the plate by two ribs showed that: 
- the maximum value of the deflection of the central point of the plate, regardless of reinforcement, is achieved in the time interval $0.001<t<0.002$. At the same time, the deflection of the central point of the unsupported plate exceeds by at least $37 \%$ the value of the deflection of the central point of the reinforced plate;

If the ribs are located at distances $d=0.4$ and $d=0.6$ from the central point, the presence of ribs leads to a decrease in the deflection value to $44 \%$. In other cases of reinforcement, the de

flection index is in the range from $37 \%$ to $44 \%$;

Calculations of deflections of the central point, supported by one, two, and four ribs of the plate showed decreasing in the maximum values of deflection, accordingly up to $12.5 \%$, $40 \%$, and $57 \%$;

- there is an abrupt change in the tangential force, and its value is the smaller, the greater the number of ribs. The reinforcement of such a plate with four annular ribs practically nullifies the action of the force except for the points of attachment of the ribs, where there are abrupt changes in the values of this force;

- shear force at the points of attachment of the ribs takes on zero values. At $r=0.3$, all cutting force curves, regardless of the presence and number of reinforcing ribs, or their absence, change their sign to the opposite. This indicates that the points of the plate with coordinate $r>0.3$ receive a deflection in the direction opposite to the deflection at $r<0.3$;

\section{References}

1. Il'in V.P., Karpov V.V. Stability of ribbed shells for large displacements, Leningrad: Stroyizdat (1986)

2. Khalmuradov R.I., Mikhailov B.K. Research of construction mechanics and reliability builds, Constructions, Moscow, pp. 153-60, (1992)

3. Khalmuradov, R.I., Karshiev A.B. Calculation of structural elements with irregularities in the regularity of the structure, Publishing House named after Abu Ali Ibn Sina, Tashkent, (2002)

4. Khalmuradov R.I. Uzbek Journal Problems of Mechanics, 6 pp. 23-32. (1995)

5. Popa C.T., Iatan R., Manescu C. Journal Proceedings in Manufacturing Systems 8 (1), pp. 53-58, (2013)

6. Ichchou MN, Berthaut J, Collet M International Journal of Solids and Structures, 45 (5), pp. 196-216, (2008), doi. org/10.1016/j.ijsolstr.2007.08.020.

7. Zhigalko Y.P., Dmitrieva L.M. Journal Research on the Theory Plates and Shells, 13, pp. 3-30, (1978)

8. Khalmuradov R.I., Yalgashev B.F., IOP Conf. Ser.: Earth Environ. Sci. 614012087 , (2020), doi: 10.1088/1755-1315/614/1/012087

9. Galiev S.U., Karshiev A.B., 1990 Strength of Materials 5 pp. 83-86.

10. Khalmuradov R.I., and Ismoilov E.A. IOP Conf IOP Conf. Ser.: Earth Environ. Sci. 614 012071, (2020) DOI: 10.1088/1755-1315/614/1/012071

11. Finnveden S.Journal of Sound and Vibration 199 (1) pp. 125-54, (1997)

12. Khudoynazarov $\mathrm{Kh}$ and Khudoyberdiyev Z.B. IOP Conf. Ser.: Earth Environ. Sci. $6140120612020 D O I: 10.1088 / 1755-1315 / 614 / 1 / 012061$.

13. Gavric L. Journal of Sound and Vibration 173 (1) pp. 113-24. (1994)

14. Khudoynazarov Kh and Yaxshiboyev Sh.R. IOP Conf. Ser.: Earth Environ. Sci. 614 012062. (2020) doi: 10.1088/1755-1315/614/1/012062.

15. Houillon L, Ichchou M.N., Jezequel L. Journal of Sound and Vibration. 281 (3-5) pp. 483-507, (2005) 
16. Mace B, The Journal of the Acoustical Society of America, 117 (5) pp. 2835-2843, (2005)

17. Benerjee S, Kundu T 2006 International Journal of Solids and Structures 43 (21) 6551-6567.

18. Popa C 2006 Al III-lea Simpozion International "Mecatronica, Microtehnologii si Materiale Noi", Targoviste. 2006.

19. Filippov I.G., Kudainazarov K. 1998 International Applied Mechanics 34 (12), pp.1204-1210. doi:10.1007/bf02700874.

20. K.Khudoynazarov et al 2021 IOP Conf. Series: Mater. Sci. Eng. 1030012098 DOI: 10.1088/1757-899X/1030/1/012098.

21. Popa C et al IOP Conf.Series: Mater. Sci. Eng. 2018. 444, 062019 DOI: 10.1088/1757-899X/444/6/062019.

22. Karpov V.V., Ignat'ev O.V., Semenov A.A. Magazine of Civil Engineering 6 pp. 147160. (2017) doi: 10.18720/MCE.74.12

23. Khudoynazarov Kh., Khalmuradov R.I., Yalgashev B.F. Bulletin of the Tomsk State University Mathematika and Mechanica, 69 139-54, doi: 10.17223/19988621/69/11

24. Filippov I.G, Kudainazarov K. 1990 Soviet Applied Mechanics. 26 (2) 161-68, (2021) doi: 10.1007/bf00887110.

25. Filippov IG., Kudainazarov K. Soviet Applied Mechanics. 26(4), pp. 351-57, (1990) DOI: $10.1007 / \mathrm{bf00887127.}$

26. Galiyev S.U. Numerical Simulation of wave processes in confined media, Kiev, (1989) 